\title{
CCD astrometry of Saturn's satellites 1990-1994
}

\author{
D. Harper ${ }^{1}$, C.D. Murray $^{1}$, K. Beurle ${ }^{1}$, I.P. Williams ${ }^{1}$, D.H.P. Jones ${ }^{1,2}$, D.B. Taylor ${ }^{2}$, and S.C. Greaves ${ }^{1}$ \\ 1 Astronomy Unit, School of Mathematical Sciences, Queen Mary and Westfield College, Mile End Road, London E1 4NS, UK \\ 2 Royal Greenwich Observatory, Madingley Road, Cambridge CB3 0EZ, UK
}

Received February 19; accepted May 15, 1996

\begin{abstract}
In this paper, we publish 1206 measurements of positions of the major satellites of Saturn made in 1990, 1991, 1993 and 1994 using CCD detectors attached to the 1-metre Jacobus Kapteyn Telescope on the island of La Palma. Analysis of the data as inter-satellite positions shows that the observations of Tethys, Dione, Rhea and Titan have root-mean-square residuals of 0. " 08 , corresponding to $500 \mathrm{~km}$ at the distance of Saturn.
\end{abstract}

Key words: satellites of saturn — astrometry

\section{Introduction}

In an earlier paper (Beurle et al. 1993), we reported on our use of a CCD camera on the 1-metre Jacobus Kapteyn Telescope (JKT) on La Palma to obtain astrometric observations of the major satellites of Saturn during a sevenday period in July 1991. These observations yielded rootmean-square (RMS) residuals of 0.10 when compared with the orbits of Harper \& Taylor (1993, 1994), demonstrating that CCDs can achieve results equal to photographic plates for this type of astrometry.

As a result of the success of the 1991 observing campaign, further sets of observations were made with the JKT in 1993 and 1994 using a CCD with twice the field of view of the CCD used in previous years. This enabled us to include both Iapetus and Phoebe in our observing programme and also to investigate new methods for astrometric calibration of the CCD.

In this paper, we publish all of our measurements of the positions of the eight major satellites made during observing campaigns in 1990, 1991, 1993 and 1994.

\section{The observing campaigns}

All of the observations listed in Tables 6-9 were obtained using the 1-metre Jacobus Kapteyn Telescope on the is-

Send offprint requests to: D. Harper

* Tables 6-9 are only available in electronic form at the CDS via anonymous ftp 130.79.128.5 land of La Palma (longitude W $17^{\circ} 53^{\prime}$, latitude N $28^{\circ} 46^{\prime}$ ) using coated CCD detectors at the $\mathrm{f} / 15$ Cassegrain focus.

We were allocated one week of telescope time in each of the years 1990, 1991, 1993 and 1994. These generally corresponded to a period around the time of Full Moon near to the date of opposition of Saturn. In the first three years, we were able to make observations throughout the entire week apart from single nights in 1991 and 1993 when poor weather or bad seeing prevailed. Additional observations were obtained during late July and early August 1990 as part of the Isaac Newton Group service programme.

During the 1994 observing run, serious forest fires broke out close to the Observatory in the early evening of August 24th, preventing operation of the telescopes during that night and eventually forcing the evacuation of the entire Observatory for several days. Consequently, observations of Saturn were obtained on only two nights in 1994.

A summary of the observing situation for all four years is given in Table 1.

Two different CCD detectors were used. In 1990 and 1991, we used a GEC chip with $22 \mu \mathrm{m}$ pixels, giving an approximate scale of 0 . 3 per pixel in the focal plane. The size of the array was $590 \times 400$ pixels. In 1993 and 1994, a larger EEV chip was available, having a $1280 \times 1180$ array of $22.5 \mu \mathrm{m}$ pixels. The corresponding fields of view are listed in Table 1. The larger field of view in 1993 and 1994 enabled us to obtain CCD frames containing images of up to eight satellites at a time. In particular, we were able to obtain images of Iapetus, the outermost satellite, in the same CCD frame as one or more of the other satellites. This is an essential aspect of our observational technique, since the exposure time of most of our CCD frames is too short (4 seconds) to record images of any astrometric reference stars and the satellite images must therefore be analysed in the form of relative positions. There are additional advantages to be gained from treating the data as intersatellite measures and these are explained in Sect. 3.3 of Harper \& Taylor (1994).

In all four years, we used a Gunn $Z$ filter with a central wavelength of $930 \mathrm{~nm}$ and a full width at half maximum of 
Table 1. Observing conditions for 1990-94. Entries in italics indicate service observations which were not part of the main campaign

\begin{tabular}{lllllll} 
Year & Observing period & $\begin{array}{c}\text { Clear } \\
\text { nights }\end{array}$ & Full Moon & $\begin{array}{l}\text { Opposition } \\
\text { of Saturn }\end{array}$ & Field of view & Observers \\
\hline 1990 & Jul 10/11 - Jul 17/18 & 8 & Jul 8 & Jul 14 & $180^{\prime \prime} \times 120^{\prime \prime}$ & IPW,DBT,DHPJ \\
& Jul 26/27 - Aug 2/3 & 8 & Aug 6 & & $180^{\prime \prime} \times 120^{\prime \prime}$ & DHPJ \\
& Aug 15/16 - Aug 17/18 & 3 & Aug 6 & & $180^{\prime \prime} \times 120^{\prime \prime}$ & DHPJ \\
1991 & Jul 4/5 - Jul 10/11 & 6 & Jul 27 & Jul 27 & $180^{\prime \prime} \times 120^{\prime \prime}$ & IPW,DBT,DHPJ \\
1993 & Aug 28/29 - Sep 3/4 & 6 & Sep 1 & Aug 20 & $380^{\prime \prime} \times 350^{\prime \prime}$ & IPW,DH,DHPJ \\
1994 & Aug 22/23 - Aug 28/29 & 2 & Aug 21 & Sep 1 & $380^{\prime \prime} \times 350^{\prime \prime}$ & DH,SCG
\end{tabular}

$150 \mathrm{~nm}$. This coincides with methane absorption bands in Saturn's atmosphere and reduces the relative brightness of the planet's globe. However, the spectrum of the rings is not dominated by methane, and the filter therefore has little effect on them. The brightness of the rings made it difficult to measure the position of Mimas reliably on many of the CCD frames since the images of the satellite were superimposed on a background of strong scattered light.

A summary of the number of images of each of the target satellites in each year is given in Table 2 .

Table 2. a) Number of images of each satellite

\begin{tabular}{lrrrrr} 
Satellite & 1990 & 1991 & 1993 & 1994 & Total \\
\hline Mimas & 41 & 16 & 9 & 7 & 73 \\
Enceladus & 103 & 47 & 28 & 21 & 199 \\
Tethys & 110 & 53 & 32 & 17 & 212 \\
Dione & 100 & 46 & 31 & 27 & 204 \\
Rhea & 114 & 49 & 32 & 30 & 225 \\
Titan & 81 & 22 & 30 & 20 & 153 \\
Hyperion & 36 & 14 & 27 & 11 & 88 \\
Iapetus & - & - & 24 & 28 & 52 \\
TOTAL & 585 & 247 & 213 & 161 & 1206
\end{tabular}

\section{Methods of calibration}

In traditional photographic astrometry, the scale and orientation of the plate are determined by measuring the positions of several reference stars whose Right Ascensions and Declinations are known accurately. The much smaller field of view of a CCD prevents this from being a viable method for calibrating such a device, and it is therefore necessary to turn to other techniques.

Several methods have been suggested by previous authors. Jones et al. (1989) used the wide double star 61
Cygni to provide a target of known position angle and separation. Colas \& Arlot (1991) proposed the use of globular clusters such as M 15 as rich fields of stars for which astrometric catalogues exist (for example, Le Campion et al. 1992). They also suggested the classical technique of star trails. We have experimented with all of these methods, and some of our findings are reported in Beurle et al. (1993).

We expected that globular clusters would provide the most reliable method of calibration. On fitting individual images of M 15 and M 92 to astrometric catalogues of these fields, we obtained root-mean-square residuals of 0.'06 from samples of 40-60 stars. However, the scale and orientation derived from the M 15 images was not consistent with those from images of M 92. This is in accord with the findings of Colas \& Arlot (1991) who were also unable to obtain reliable calibration parameters from their reduction of images of M 15. They suggested that the main cause of the discrepancy is probably the flexure of the telescope as it was moved from one target to another.

We are currently working on a simplified empirical model which assumes the flexure to be a function of the target's position (Jones 1996). We have not made use of calibration parameters from globular cluster images whilst preparing our observations for publication in this paper.

Colas \& Arlot (1991) describe several reductions of their observations of the Martian satellites in which the scale and orientation of the CCD device during each night are determined by calculating the values which minimise the observed-minus-computed (O-C) residuals of the inter-satellite positions when compared to the theories of Chapront-Touzé (1989). We have reported the use of a similar technique when analysing our 1991 observations (Beurle et al. 1993). In that paper, we calculated a correction to the scale factor which would optimise the $\mathrm{O}-\mathrm{C}$ residuals when compared to the orbital models of Harper \& Taylor $(1993,1994)$.

During the 1990 campaign, no usable calibration images were obtained. Moreover, it is not clear whether images of 61 Cygni, M 15 and M 92 obtained in later 
campaigns will prove to be of any value in providing consistent scale and orientation parameters. Consequently, we have adopted the technique described by Colas and Arlot.

Table 2. b) Number of satellites per CCD frame

\begin{tabular}{lrrrr} 
Number of satellites & \multicolumn{5}{c}{ Number of frames } \\
in frame & 1990 & 1991 & 1993 & 1994 \\
\hline 2 & 32 & 4 & 3 & 3 \\
3 & 25 & 10 & 1 & 1 \\
4 & 46 & 18 & - & 8 \\
5 & 32 & 19 & 3 & 7 \\
6 & 17 & 7 & 10 & 13 \\
7 & - & - & 15 & 1 \\
8 & - & - & 3 & -
\end{tabular}

In our implementation of this method, we took approximate values of the scale of the CCD from images of 61 Cygni or one of the globular clusters. In all years, we assumed initially that one axis of the CCD was parallel to the direction to the true North celestial pole. We then employed an iterative least-squares process to determine small corrections to the scale and orientation which optimised the goodness of fit between the observed positions of the major satellites and the corresponding positions predicted using the orbital models of Harper \& Taylor (1993, 1994). Six such sets of corrections were calculated: one for each main campaign, with separate sets for the observations made during the periods 26 July to 3 August and 15 to 17 August 1990. The CCD was dismounted from the telescope between 4 and 14 August.

The observations were processed as inter-satellite measurements because the globe of the planet is saturated in all of the CCD images and its centre cannot reliably be determined. Such inter-satellite measurements are affected by differential parallax, aberration and refraction. The first two effects are small, but differential refraction can introduce changes of up to one part in $10^{3}$ in relative positions when the satellites are observed at elevations of less than $40^{\circ}$ above the horizon; this is equivalent to $0^{\prime \prime} \cdot 3$ in extreme cases. All three effects were incorporated into the positions derived from the orbital models, and these positions could therefore be compared directly to the raw observed positions.

Only observations of Tethys, Dione, Rhea and Titan were used in calibrating the CCD, as these satellites have the most reliable orbital theories.

\section{Format of the observations}

The data in this paper are published in the form of raw pixel coordinates which were determined by the centrefinding tool of the IRAF (Image Reduction and Analysis
Facility) software. Publication of the data in this format will enable future investigators to make direct use of the original CCD images if they so wish.

We use $\xi$ to denote the column coordinate and $\eta$ to denote the row coordinate in the CCD array. In the data tables, the satellite positions are given as $(\xi, \eta)$ pairs.

The orientation of the CCD device changes from one year to another, depending on how the device was mounted by the Observatory staff. However, one axis of the device is always aligned roughly north-south. We therefore use $x$ to denote a direction parallel to the axis of the CCD which is nearest to the east-west direction, with $x$ increasing eastwards (i.e. in the direction of increasing Right Ascension). Likewise, $y$ is parallel to the axis of the CCD which is nearest to the north-south direction, with $y$ increasing northwards (i.e. in the direction of increasing Declination).

Table 3 shows the approximate directions of the CCD coordinate axes in each year, together with the transformation from $(\xi, \eta)$ to $(x, y)$.

Table 3. Orientation of the CCD device and transformation from $(\xi, \eta)$ coordinates to $(x, y)$

\begin{tabular}{ccccc} 
Year & \multicolumn{2}{c}{ Axes of CCD } & \multicolumn{2}{c}{ Transformation } \\
& $\xi$ & $\eta$ & $\Delta x$ & $\Delta y$ \\
\hline 1990 & North & West & $-\Delta \eta$ & $+\Delta \xi$ \\
1991 & South & East & $+\Delta \eta$ & $-\Delta \xi$ \\
1993 & East & South & $+\Delta \xi$ & $-\Delta \eta$ \\
1994 & East & South & $+\Delta \xi$ & $-\Delta \eta$
\end{tabular}

Table 4. Calibration parameters for the observations

\begin{tabular}{lcc} 
Dataset & $\delta P($ degrees $)$ & $\rho(\operatorname{arcsec} /$ pixel $)$ \\
\hline $1990 \mathrm{a}$ & $+1.1746 \pm 0.0030$ & $0.302844 \pm 0.000016$ \\
$1990 \mathrm{~b}$ & $+1.2089 \pm 0.0066$ & $0.302802 \pm 0.000035$ \\
$1990 \mathrm{c}$ & $+1.0900 \pm 0.0070$ & $0.302902 \pm 0.000037$ \\
1991 & $+0.2682 \pm 0.0050$ & $0.302936 \pm 0.000027$ \\
1993 & $+2.4525 \pm 0.0028$ & $0.309693 \pm 0.000015$ \\
1994 & $+0.0959 \pm 0.0032$ & $0.309801 \pm 0.000017$
\end{tabular}

Let $(\Delta \xi, \Delta \eta)$ denote the position of satellite $\mathrm{B}$ with respect to satellite $\mathrm{A}$ in raw pixel coordinates. Further, let $\rho$ denote the scale of the CCD device in units of arcseconds per pixel. This assumes that the pixels are square; we have run calibration trials with different scales in the $\xi$ and $\eta$ directions but have found no significant difference 
Table 5. Example transformations from pixel coordinates to true equator and equinox of date

\begin{tabular}{|c|c|c|c|c|c|c|c|c|}
\hline Date & Sat. A & Sat. B & $\Delta \xi$ & $\Delta \eta$ & $\Delta x$ & $\Delta y$ & $\Delta \alpha \cos \delta$ & $\Delta \delta$ \\
\hline 1990 July 10.0 & Rhea & Dion & +88.24 & +332.40 & -332.40 & +88.24 & $-100^{\prime \prime} 10$ & $+28^{\prime \prime}$. \\
\hline 1991 July 4.991003 & Rhea & Dione & +144.38 & +305.74 & +305.74 & -144.38 & $+92 . .41$ & $-44^{\prime \prime} .17$ \\
\hline 1993 Aug. 28.894738 & Rhea & Dione & +273.71 & +67.02 & +273.71 & -67.02 & $+83^{\prime \prime} .80$ & $-24^{\prime \prime} \cdot 36$ \\
\hline 1994 Aug. 22.939692 & Titan & Rhea & +782.25 & +28.89 & +782.25 & -28.89 & $+242^{\prime \prime} 33$ & $-9^{\prime \prime} 36$ \\
\hline
\end{tabular}

between the two scales. Let $\delta P$ denote the position angle of the north-south axis of the CCD with respect to the true pole of date, measured positive towards the East.

First calculate $\Delta x$ and $\Delta y$ according to Table 3 . Then the differential coordinates $\Delta \alpha \cos \delta$ and $\Delta \delta$ referred to the true equator and equinox of date are calculated from

$$
\left(\begin{array}{c}
\Delta \alpha \cos \delta \\
\Delta \delta
\end{array}\right)=\rho \cdot\left(\begin{array}{cc}
\cos \delta P & \sin \delta P \\
-\sin \delta P & \cos \delta P
\end{array}\right)\left(\begin{array}{c}
\Delta x \\
\Delta y
\end{array}\right)
$$

\subsection{Values of $\delta P$ and $\rho$}

The parameters $\rho$ and $\delta P$ have been determined empirically for each observing campaign by comparing the observations with the orbital theories of Harper \& Taylor $(1993,1994)$ and determining the values of $\rho$ and $\delta P$ which yield the best fit between the observations and the theories. Work is in progress to obtain independent values of these parameters from observations of globular clusters and other fiducial star fields (Jones 1996).

The values which we have adopted at present are given in Table 4. They are based upon measurements of the positions of Tethys, Dione, Rhea and Titan.

The 1990 data has been divided into three subsets. Set (a) covers 10-18 July and represents the main observing programme in that year. Set (b) covers 26 July to 3 August, whilst set (c) covers 15-17 August. The CCD detector was removed between sets (b) and (c) while a different instrument was in use. In addition, it is not possible to guarantee that the CCD was not rotated between sets (a) and (b); the difference in the $\delta P$ values for these sets is 0.0342 , which is eleven times the standard error of set (a), suggesting that some alteration of the alignment may have occurred.

\subsection{Example calculation}

In order to assist the reader in checking the validity of the formulae, we present four sample calculations in Table 5. In each case, the example is taken from the first image listed for the given year.

\section{The observations}

In Tables 6-9, we give the observed positions of the satellites in the form of pixel coordinates, as described in the previous section. These are apparent topocentric data; no correction has been made for the effects of refraction, stellar aberration or parallax. The date and time of the middle of the exposure are given in Universal Time. The exposure time is also given. The satellites are listed in the conventional order of increasing semi-major axis.

Table 6. Observations made in 1990

This table is only available in electronic form at the CDS via anonymous ftp.

Table 7. Observations made in 1991

This table is only available in electronic form at the CDS via anonymous ftp.

Table 8. Observations made in 1993

This table is only available in electronic form at the CDS via anonymous ftp.

Table 9. Observations made in 1994

This table is only available in electronic form at the CDS via anonymous ftp.

\section{Analysis of the observations}

We have calculated the root-mean-square residuals of the observations in the form of inter-satellite $\Delta \alpha \cos \delta$ and $\Delta \delta$ measures. The orbital models are those of Harper \& Taylor $(1993,1994)$. In Table 10 we present the statistics for Tethys, Dione, Rhea and Titan. The rejection level was $1^{\prime \prime} .0$ and this table is directly comparable with Table $3 \mathrm{~b}$ in Harper \& Taylor (1994). For comparison, we give the same statistics for the top five datasets listed in that paper. 
Table 10. Statistics of observed-minus-computed residuals for observations of Tethys, Dione, Rhea and Titan. Type code "C 1 " denotes $\Delta \alpha \cos \delta$ and $\Delta \delta$ measures from CCD images. $N_{\mathrm{U}}$ is the number of observations included in the solution. $N_{\mathrm{T}}$ is the total number of observations in the dataset. $\mu$ is the mean residual and $\sigma$ is the standard deviation about the mean. See Strugnell \& Taylor (1990)

\begin{tabular}{|c|c|c|c|c|c|c|c|c|c|c|}
\hline \multirow{2}{*}{$\begin{array}{l}\text { Ref. } \\
\text { No. }\end{array}$} & \multirow[t]{2}{*}{ Data Set } & \multirow[t]{2}{*}{ Type } & \multicolumn{4}{|c|}{$\Delta \alpha \cos \delta$} & \multicolumn{4}{|c|}{$\Delta \delta$} \\
\hline & & & $N_{\mathrm{U}}$ & $N_{\mathrm{T}}$ & $\begin{array}{c}\mu \\
\text { Arcseconds }\end{array}$ & $\sigma$ & $N_{\mathrm{U}}$ & $N_{\mathrm{T}}$ & $\begin{array}{c}\mu \\
\text { Arcseconds }\end{array}$ & $\sigma$ \\
\hline 90 & LaPalma CCD (1990a) f & $C 1$ & 333 & 333 & $-0.001 \pm 0.003$ & 0.06 & 333 & 333 & $-0.003 \pm 0.005$ & 0.09 \\
\hline 91 & f LaPalma CCD (1990b) & $C 1$ & 54 & 54 & $-0.009 \pm 0.008$ & 0.06 & 54 & 54 & $0.006 \pm 0.012$ & 0.09 \\
\hline 92 & f LaPalma CCD (1990c) & $C 1$ & 37 & 37 & $0.010 \pm 0.009$ & 0.06 & 37 & 37 & $-0.009 \pm 0.008$ & 0.05 \\
\hline 97 & f LaPalma CCD (1991) & $C 1$ & 184 & 184 & $-0.003 \pm 0.006$ & 0.08 & 184 & 184 & $0.000 \pm 0.008$ & 0.11 \\
\hline 98 & f LaPalma CCD (1993) & $C 1$ & 177 & 177 & $-0.028 \pm 0.006$ & 0.08 & 177 & 177 & $0.014 \pm 0.006$ & 0.08 \\
\hline 99 & f LaPalma CCD (1994) & $C 1$ & 106 & 106 & $0.000 \pm 0.008$ & 0.08 & 106 & 106 & $-0.007 \pm 0.008$ & 0.09 \\
\hline 10 & Alden \& O'Connell (1928) f & $P 1$ & 300 & 303 & $-0.033 \pm 0.005$ & 0.09 & 301 & 303 & $0.003 \pm 0.004$ & 0.07 \\
\hline 33 & Tolbin (1985) f & $P 0$ & 168 & 168 & $0.009 \pm 0.006$ & 0.08 & 167 & 168 & $0.005 \pm 0.010$ & 0.13 \\
\hline 48 & Veillet \& Dourneau (1992) f & $P 1$ & 434 & 434 & $-0.007 \pm 0.006$ & 0.13 & 434 & 434 & $0.012 \pm 0.005$ & 0.10 \\
\hline 31 & Pascu (1982) f & $P 1$ & 762 & 770 & $0.000 \pm 0.005$ & 0.12 & 763 & 770 & $-0.004 \pm 0.004$ & 0.10 \\
\hline 47 & Veillet \& Dourneau (1992) f & $P 1$ & 73 & 73 & $-0.010 \pm 0.017$ & 0.14 & 73 & 73 & $-0.032 \pm 0.009$ & 0.08 \\
\hline
\end{tabular}

\section{Conclusions}

This paper lists over 1200 observations of the major satellites of Saturn made during the apparitions of 1990-94 using CCD detectors on the Jacobus Kapteyn Telescope. As far as we are aware, these are the only such observations which have been obtained during the period, and they are therefore a valuable resource to anyone wishing to investigate the orbits of the satellites.

The relative ease with which such high-precision observations can be obtained demonstrates the value of CCD astrometry applied to the satellites of the major planets. In particular, the use of the satellites themselves to calibrate the scale and orientation of the CCD further simplifies the reduction of the observations. However, additional work needs to be done in order to validate this technique, since it is possible that it may act to perpetuate the scale errors that are known to exist in the semi-major axes of the orbits derived from pre-1966 visual observations.

Acknowledgements. The Jacobus Kapteyn Telescope is operated on the island of La Palma by the Royal Greenwich Observatory in the Spanish Observatorio del Roque de los Muchachos of the Instituto de Astrofisica de Canarias. The authors wish to thank the staff of the observatory for their kind assistance. We also thank Dr. R.A. Jacobson of JPL for carrying out an independent analysis of the residuals of the observations and helping to identify a small number of inconsistencies in the original data set. We are grateful to the referee, Dr. Gérard Dourneau, for helpful and constructive criticism of the original version of this paper. This work was carried out with financial support from the U.K. Particle Physics and Astronomy Research Council; one of the authors (CDM) wishes to thank the PPARC for the award of an Advanced Fellowship. S.C. Greaves is funded by a PPARC research studentship.

\section{References}

Alden H.L., 1929, AJ 40, 88

Alden H.L., O'Connell W.C., 1928, AJ 38, 53

Beurle K., Harper D., Jones D.H.P., Murray C.D., Taylor D.B., Williams I.P., 1993, A\&A 269, 564

Chapront-Touzé, 1989, A\&A 240, 159

Colas P., Arlot J.-E., 1991, A\&A 252, 402

Harper D., Taylor D.B., 1993, A\&A 268, 326

Harper D., Taylor D.B., 1994, A\&A 284, 619

Jones D.H.P., Sinclair A.T., Williams I.P., 1989, MNRAS 237, $15 \mathrm{P}$

Jones D.H.P., 1996, in Proceedings of I.A.U. Symposium 172, held in Paris, 3-8 July 1995 (to be published)

Le Campion J.-F., Geffert M., Dulou M.R., Colin J., 1992, A\&AS 95, 233

Pascu D., 1982, data held at the U.S. Naval Observatory, Washington, D.C.

Strugnell P.R., Taylor D.B., 1990, A\&AS 83, 289

Tolbin S.V., 1985, Glav. Astron. Obs. Navk. Leningrad, p. 14

Veillet C., Dourneau G., 1992, A\&AS 94, 291 